\title{
Treatment of overweight and obesity during and after a pandemic. Let's not wait for the development of complications - new guidelines for doctors
}

\author{
Guidelines developed by Experts endorsed by the Polish Association \\ for the Study of Obesity, Polish Psychiatric Association, Polish Society of Hypertension, Scientific \\ Section of Telepsychiatry of the Polish Psychiatric Association, \\ Polish Association of Cardiodiabetology, Polish Association of Endocrinology, \\ and The College of Family Physicians in Poland
}

Social patronage of the Foundation for People with Obesity OD-WAGA

\begin{abstract}
Magdalena Olszanecka-Glinianowicz', Dominika Dudek², Krzysztof J. Filipiak³, Marek Krzystanek, Leszek Markuszewski ${ }^{5}$, Marek Ruchała', Elżbieta Tomiak ${ }^{7}$

'Health Promotion and Obesity Management Unit, Department of Pathophysiology, Medical Faculty in Katowice, Medical University of Silesia, Polish Association for the Study of Obesity

${ }^{2}$ Department of Psychiatry, Collegium Medicum, Jagiellonian University in Cracow, Polish Psychiatric Association

${ }^{3} 1^{\text {st }}$ Department and Clinic of Cardiology, Medical University of Warsaw, Polish Association of Hypertension

${ }^{4}$ Department of Psychiatric Rehabilitation, Medical Faculty in Katowice, Medical University of Silesia, Scientific Section of Telepsychiatry of the Polish Psychiatric Association

${ }^{5}$ Centre for Heart and Vascular Diseases, Internal and Metabolic Diseases, Faculty of Medical Sciences and Health Sciences,

University of Humanities and Technology in Radom, Polish Association of Cardiodiabetology

${ }^{6}$ Department and Clinic of Endocrinology, Metabolism and Internal Diseases, Poznań University of Medical Sciences, Polish Association of Endocrinology

'VITA — Group of Family Doctors in Otyń, The College of Family Physicians in Poland
\end{abstract}

Reprinted with kind permission from: Nutrition, Obesity and Metabolic Surgery 2020; 6, 2: 1-13.

\begin{abstract}
The treatment of obesity in the pandemic era has become more important than ever. The current situation is conducive to the worsening of disease and the development of new diseases, mainly as a result of compensating negative emotions with food. Taking into account the data on the impact of obesity and its complications on the severity of the course and the risk of death due to COVID-19, we recommend using the 2016 American Endocrine Society's criteria for the diagnosis of obesity instead of the $1998 \mathrm{WHO}$ criteria. We also recommend diagnosing eating under the influence of emotions and the occurrence of eating disturbances, such as compulsive eating syndrome, night eating syndrome and food addiction, and complications of obesity, in any person with a BMI $\geq 25 \mathrm{~kg} / \mathrm{m}^{2}$.
\end{abstract}

Address for correspondence: Prof. Magdalena Olszanecka-Glinianowicz, MD, PhD, Health Promotion and Obesity Management Unit, Department of Pathophysiology, Medykow Street 18, 40-752 Katowice, Poland; e-mail: magolsza@gmail.com 
The approach to treatment should be individualised and should not be limited to nutritional and physical activity education alone. Each patient should be offered appropriately selected pharmacotherapy, and, if necessary, also psychotherapy. The first-line drug should be a combined preparation containing naltrexone and bupropion (Mysimba ${ }^{\circ}$ ). Liraglutide in a dose of $3 \mathrm{mg}$ (Saxenda ${ }^{\circ}$ ) should be considered as a second-line drug in a situation where eating under the influence of emotions is excluded (reaching for food in situations of experiencing negative and positive emotions and boredom, eating disturbances: compulsive eating syndrome, night eating syndrome, and food addiction) and depressed mood or there are permanent contraindications to the use of the first-line drug.

It is unethical not to treat obesity or refer the patient to another doctor for treatment. The use of telemedicine tools can facilitate work in therapeutic teams (doctor, dietitian, psychotherapist), as well as improve patient compliance with pharmacotherapy and changes in eating habits and the level of physical activity recommendations.

Key words: obesity treatment; pharmacotherapy; telemedicine; guidelines for doctors

Arterial Hypertens. 2020, vol. 24, no. 3, pages: 93-105

DOI: $10.5603 /$ AH.a2020.0019

\section{Preamble}

Obesity has been recognised as a disease by the World Health Organisation (WHO) for many years and is included in the International Classification of Diseases (ICD-10) under number E.66.

The WHO defines obesity as abnormal or excessive accumulation of fat in adipose tissue causing deterioration of health. The health consequences of obesity depend on the degree of excess body fat, its distribution, and the duration of obesity. It is a chronic disease without a tendency to spontaneously resolve, but with a tendency to relapse. It is not a metabolic disease. It should be noted that, it is a disease of a complex aetiology in which various causative factors lead to eating disturbances that cannot be counteracted by physical activity, resulting in a positive energy balance and the storage of excess energy in adipose tissue. Increasingly, obesity is classified as a psychosomatic disease.

Overweight is a condition defined as pre-obesity, in which the degree of excess fat that does not yet meet the criteria for diagnosing obesity [1].

Contrary to popular belief, obesity is not the fault of the patient. Blaming the patient for being ill is not ethical and leads to a lack of effective treatment, and as a consequence the disease progresses, its complications develop, the quality of life deteriorates, disability develops, and life expectancy shortens [2].

The contemporary approach to making therapeutic decisions is based on the principles of biomedical ethics published in the late 1970s, including patient autonomy, benefits and safety, and fairness.

How the autonomy of a patient with obesity is violated:

- Lack of access to an appropriate obesity treatment system and reimbursement of drugs supporting its treatment, as well as specialist medical advice, dietary advice, and psychotherapy.

- The archaic and pejorative view that obesity is the result of a lack of discipline and self-control, also among healthcare workers. This stigmatises obese patients, influences their patient judgment, interpersonal behaviour, and treatment decisions.

- Patients' negative experiences from previous contacts with healthcare professionals lead to stress and avoidance of seeking care, distrust of doctors, and non-compliance with their recommendations.

It should be emphasised that stigma and prejudices restricting access to appropriate treatment are an unacceptable violation of the patient's right to autonomy.

Ethical benefit and security are based on the principle "Do No Harm First". In this context, there are two dimensions: individual and social. In the individual dimension, the ethical aspect includes not recommending methods of diagnosing and treating obesity, the effectiveness and safety of which have been scientifically proven, and not informing the patient about all methods that can and should be applied to him/her. However, in social terms, there is a tendency to blame obese patients for the fact that their excess body mass results in a cost to other members of society. Interestingly, patients with complications of obesity are not blamed for the fact that they suffer from them, which creates a paradox, e.g. you are guilty that you suffer from obesity, but it is not your fault that you suffer from one of its most common complications: type 2 diabetes. It is unethical to blame obese patients for exposing other members of society to costs because they are not provided with appropriate medical care in accordance with current knowledge. 
Justice in terms of etical includes resource constraint, respect for individual rights, and adherence to established laws.

Patients diagnosed with obesity experience inequalities in health and limitations in self-determination not only because of the underlying disease, but also when they develop other chronic diseases due to the lack of equipment and negative attitudes of medical staff caused by stereotypical thinking, and lack of knowledge in the field of obesity treatment and inability to refer the patient to an obesity treatment centre $[2,3]$.

The principles of an ethical approach to obesity, developed by the American Society of Gynaecologists and Obstetricians, show that negative attitudes towards obese patients occur even among doctors specialising in obesity treatment. The conducted studies show that the reasons for this negative attitude are very different from the fact that the doctor also suffers from obesity to the aforementioned blaming patients for their disease, perceiving them as problematic and time-wasting. It has been observed that this results in a disturbance of the doctor-patient relationship because doctors show less empathy towards obese patients, are less likely to engage in counselling and patient-centred care, and paradoxically spend less time on them. Such an attitude of a doctor may delay the proper diagnosis of the cause of obesity and initiate effective treatment. Moreover, a patient who has experienced prejudice or stigmatisation may avoid treatment not only of obesity, but also of its complications. It has been shown, inter alia, that neoplasms in obese patients are diagnosed at a high degree of advancement because they appear not much less frequently for screening $[2,4]$.

Not only from a medical but also ethical point of view, every doctor should perceive obesity as a chronic disease with a complex etiology that requires treatment. A patient with obesity should be treated with respect, and his/her illness should not be a source of shame and self-blame. A physician should use appropriate medical vocabulary in relation to an obese patient, show empathy towards him/her and give advice appropriate to his/her situation, as well as implement all possible therapeutic procedures, including pharmacotherapy and psychotherapy [2]. The principle of person-centred care should be the norm in the approach to obese patients [5].

\section{Why should obesity be treated during a pandemic?}

In Poland, nearly 8 million adults suffer from obesity diagnosed on the basis of the World Health Organi- sation criteria, and overweight is diagnosed in almost 19 million. Due to the lack of reliable data, estimating the incidence of all chronic obesity complications is extremely difficult. Numerous data confirm that the causal treatment of obesity complications is its effective treatment with the use of all scientifically proven methods, tailored to the individual patient's needs.

The COVID-19 pandemic has proven that the current approach, focused mainly on symptomatic treatment of obesity complications, does not bring the intended results if the patient becomes infected. The inequality in health experienced by obese patients has made them the most vulnerable to severe COVID-19 infection and death thereof.

Data from China, Italy, and the USA showed that obese patients infected with COVID-19 had a longer duration of infection and more often required intensive medical supervision, including intubation and mechanical ventilation. Moreover, obesity and its complications were independent risk factors for hospitalisation and death of the infected [6-9]. It should also be noted that data from a study conducted in New York showed that obesity and its complications caused severe COVID-19 infection and increased the risk of death also among children and adolescents [10].

Overweight in patients with SARS-COV-2 infection increased the risk of developing severe pneumonia by $86 \%$, and obesity by $142 \%$ [11]. What are the reasons for this? Obese patients have decreased immunity (chronic systemic inflammatory reaction, increased activation of the hypothalamic-pituitaryadrenal system, low physical activity), they often have obese hypoventilation syndrome (reduced chest volume - disorders of ventilation and perfusion), left ventricular hypertrophy, and sometimes heart failure.

The major complications of obesity also increase the risk of death from COVID-19 (hypertension by $6 \%$, type 2 diabetes by $7.3 \%$, cardiovascular disease by $10.5 \%$, chronic lung disease by $6.3 \%$, and cancer by $5.6 \%)[12]$.

It should be emphasised that quarantine in the long term due to exposure to stress may increase people's susceptibility to obesity development, a risk factor for severe COVID-19 infection [13, 14]. It should also be added that the same stressors may have an impact on people not subject to forced quarantine. Food is an easily accessible "stepping stone" from problems; therefore, we are dealing with an increase in the percentage of people who eat under the influence of emotions and those who meet the criteria for the diagnosis of eating disturbances (compul- 
sive eating syndrome, night eating syndrome, food addiction) and those with worsening of pre-existing disturbances.

Factors that cause anxiety and sadness at present include the following: adaptation to new functioning conditions, the possibility of infection, illness of relatives - especially the elderly, fear of creating a threat to others, uncertain future in the health and social dimension (it is not known when the pandemic will end and when life will return to normal, it is not known whether the pandemic will not return in the future, fear of what the world will look like after the pandemic, how much we will have to change our lifestyle, our habits, and re-evaluate our dreams, fear of worsening economic situation, loss of job, and collapse financial), social isolation and increasing family conflicts, organisational problems related to remote work and remote learning, limitations in relieving stress outside the home (fear of using fitness clubs, cinemas, theatres, and other entertainment venues, and traveling). Additionally, there is a significant deficit of positive stimuli.

A separate factor influencing weight gain during the pandemic is the reduction in physical activity due to the introduced restrictions and quarantine.

\section{The reward system and the regulation of food intake}

The regulation of food intake is a complex process and is not limited to satiety and hunger, which are biological signals controlled by the hypothalamus. Hunger is a physiological feeling of the state of higher organisms related to a lack of food. It is also a contributing impulse to food intake and gaining behaviour. However, satiety inhibits this impulse.

An important role in human food intake is played by the sensation of appetite, which causes the search for a specific food not to satisfy hunger but to feel the pleasure of eating it. Appetite is driven by emotions and is independent of the feeling of satiety. The reward system is responsible for the feeling of appetite $[15,16]$.

Even in people with normal body mass, stress (the body's reaction to events that disrupt its homeostasis - stressors related to experiencing both negative and positive emotions that exceed our ability to cope effectively) and the associated activation of the hypothalamus-pituitary-adrenal gland axis increases the release of cortisol, reduces the sensitivity of the hypothalamus to the action of leptin, and enhances the influence of the reward system, which is responsible for food intake related not to biological need but to pleasure [17]. Activation of the reward system, including its parts such as the nucleus accumbent, increases the tendency to eat high-energy food high in sugar, fat, and sodium [18]. Stress can lead to eating more food, and with a higher propensity to eat high-energy foods. In addition, stress reduces the tendency to exercise and interfere with sleep. The mechanism of treating food as a reward or consolation arises in infancy or may be established later in life. Overeating can be a way to reduce anxiety and frustration. Many people eat food when the stress factor is unclear and causes a predominant feeling of anxiety. Eating becomes a way to regain self-control over the level of feeling emotions.

Stimulation of dopamine neurons in the ventral tegmental area causes the release of dopamine in the nucleus accumbens. Activation of dopamine receptors causes a feeling of conscious pleasure (prefrontal cortex) and motor activity related to the search for reward (basal ganglia). The hippocampus (memory) and amygdala (emotions) are also activated. The subjective feeling of pleasure is proportional to the amount of dopamine released from the nerve endings in the nucleus accumbens. Tasty food increases dopamine levels by $50 \%$. It can be concluded that reducing dopamine secretion in the structures of the reward system causes the desire/impulse to obtain pleasure, and endogenous opioids increase the feeling of pleasure from, for example, eating [19].

\section{Diagnosis of obesity - should the new criteria be used?}

The 1998 diagnostic criteria for overweight and obesity are based solely on body mass index (BMI) values and do not take into account the amount or distribution of adipose tissue in the body or their impact on the patient's health [1]. Data on the impact of overweight and obesity and their complications on the severity of the course and the risk of death from COVID-19 indicate that it is time to revise these archaic criteria and adopt algorithms that can be used in any doctor's office, but taking into account the patient's general health. These conditions are met by the criteria proposed in 2016 by the American Endocrine Societies. On the basis of which we recognise the following:

- overweight degree 0 - BMI $25.0-29.9 \mathrm{~kg} / \mathrm{m}^{2}$ and without complications such as the following: pre-diabetes, type 2 diabetes, dyslipidaemia, arterial hypertension, cardiovascular disease, nonalcoholic fatty liver disease, polycystic ovary syndrome, female infertility, male hypogonadism, 
asthma, sleep apnoea syndrome, hypoventilation syndrome, gastroesophageal reflux disease, stress urinary incontinence, osteoarthritis, and depression,

- obesity degree $0-\mathrm{BMI} \geq 30 \mathrm{~kg} / \mathrm{m}^{2}$ and without the above-mentioned complications,

- first-degree obesity - BMI $\geq 25 \mathrm{~kg} / \mathrm{m}^{2}$ and $\geq 1$ mild or moderate complication,

- second-degree obesity - BMI $\geq 25 \mathrm{~kg} / \mathrm{m}^{2}$ and $\geq 1$ severe complication [20].

These criteria allow for a much earlier diagnosis of obesity and initiation of treatment, which may significantly improve health or prevent the development of complications. Therefore, we recommend using them.

However, these criteria also do not include the diagnosis of the root cause of the positive energy balance. Proper diagnosis of the cause of obesity development allows the selection of adequate treatment methods and increases its effectiveness. Most of the failures in the treatment of obesity are the result of the wrong approach that proper nutritional education and the strong will of the patient will suffice. The lack of understanding of the role of emotions in influencing food intake and the fact that disorders in the functioning of the reward system make the patient "willing but unable" frustrate both the doctor and the patient, which results in discouragement from undertaking obesity treatment. The view that eating disorders should be diagnosed by a psychiatrist is incorrect because screening is performed using simple diagnostic tools, such as diagnostic criteria:

- compulsive eating syndrome: repeated episodes of uninhibited eating at least once a week for three months and at least three of the following symptoms: eating much faster than normal, eating until you feel uncomfortably full, eating large portions of food without feeling physically hungry, eating alone due to shame/embarrassment in eating, feeling disgusted with oneself, depression or guilt after overeating, as well as a pronounced suffering about the way of eating and the lack of compensatory activities related to it (induction of vomiting, use of diuretics, significant increase in physical activity) [21];

- night eating syndrome: eating $\geq 25 \%$ of one's daily amount of food after the evening meal or at night with consciousness at least twice a week for at least three months and at least three of the following symptoms: skipping breakfast due to lack of appetite at least four times a week, a strong need to eat between the evening meal and falling asleep or at night, difficulty falling asleep or waking up from sleep at least four nights a week, the conviction that food is needed as a condition for starting or returning to sleep, frequent worsening of mood in the evening, as well as significant suffering or deterioration in functioning, and the lack of criteria for mental bulimia and seizure syndrome overeating [22];

- food addiction: eating more, or more than intended, persistent desire to eat or unsuccessful attempts to limit food consumption, devoting a large amount of time to eating activities, neglecting social duties and activities, eating food despite negative physical, mental, and social consequences, limiting or abandoning important social, professional, or recreational activities due to food consumption, and the occurrence of withdrawal syndrome [21].

Any doctor should treat eating disorders in cooperation with a psychotherapist. However, it should be remembered that eating under the influence of emotions does not have to meet the criteria for the diagnosis of eating disorders, and it occurs in an even greater group of obese patients than the disorders discussed above. Therefore, patients should be asked about eating under stress, after a stressful situation, in conditions of boredom, or even strong positive emotions.

\section{Obesity treatment - who, when, and how?}

The ideal situation would be if an obese patient could be referred to a specialist centre, where he/she would be looked after by an obesitologist in cooperation with a dietitian, psychologist, and physiotherapist. Because there is no coordinated system of obesity treatment in Poland, and its treatment is among the duties of a family doctor and specialist doctors caring for patients diagnosed with obesity complications, it is these groups of doctors who are obliged to diagnose and treat this disease. If the doctor is unable or unwilling to treat obesity, after diagnosing it, he/ /she should refer the patient to another doctor who will take care of the patient. We suggest that patients be referred to doctors who have obtained certificates of the Polish Association for the Study of Obesity. The list of such people is available on the websites ptbo.edu.pl or certyfikacjaptbo.pl. [23]. It is unethical not to diagnose and treat obesity or to refer the patient to another physician to treat obesity. Obesity is a disease, and referring a patient to a dietitian as the only action without implementing all the necessary therapeutic methods is also an unethical action.

It should be emphasised that four out of five medications registered in the USA for the treatment 
of obesity and two out of every three approved in Europe act in the central nervous system. The only drug that acts locally in the gastrointestinal tract is orlistat, a drug of marginal importance in the pharmacological treatment of obesity. Drugs influencing food intake have a pharmacological effect in the CNS, because these are the centres responsible for the feeling of satiety, hunger, and appetite. However, this does not provide sufficient justification for the drugs registered to support obesity treatment to be prescribed only by a psychiatrist, and even less so that only a psychiatrist can diagnose eating disturbances

Obesity is a progressive disease, and the development of its complications depends on the distribution of adipose tissue, the stage of the disease, and its duration. In order to prevent the progression of the disease and the development of complications, treatment of obesity should be started as early as possible at the stage of overweight degree 0 . It should also be undertaken at any other stage of the disease, without waiting for the development of complications.

The overarching goal of obesity treatment is to inhibit the progression of the disease, avoid recurrences, prevent the development of complications caused by excess body fat or reduce their severity, improve the overall health and quality of life of the patient, and extend their life. To achieve this goal, different percentages of body mass reduction from baseline may be needed in different patients [23].

In order to prevent obesity complications, the initial goal is a reduction of $5-10 \%$ of the initial body mass over a 3-6-month period, then the same maintenance period, followed by another $5-10 \%$ reduction in body mass. The main goal in patients without obesity complications is to reduce the severity of the disease by one degree. On the other hand, in patients with complications, the overriding goal is to achieve a weight reduction that will significantly improve the control of this complication, the possibility of reducing the number or doses of drugs used due to the complication or discontinuing their use, and in some cases achieving remission in terms of the complication [23].

In the treatment of obesity, unlike slimming, the goal is not to lose weight quickly and significantly, but to reduce it slowly and over the long term, which will improve health and quality of life, and increase life expectancy. Therefore, the methods used in the treatment of obesity, including pharmacotherapy, are to cause a slow loss of body mass. This should be carefully explained to the patient, because the result may not match their expectations, which must be verified, and the new goal must be accepted by the patient. The goal assigned to the patient must be specific, measurable, acceptable, realistic, and timely (SMART). On the way to the final goal, it is necessary to set sub-goals and short-term goals ranging from three to six months [23].

As already mentioned, the selection of obesity treatment methods must be individualised, taking into account, first of all, the root cause of a disturbed energy balance. It should be remembered that this treatment is a long-term process.

In the treatment of overweight and obesity, the doctor should follow the $5 \mathrm{~A}$ rule:

- ASK - explaining to the patient the essence of the disease and its consequences and assessing his/her readiness to change,

- ASSESS - assessment of health, causes of weight gain, and the occurrence of complications caused by excess fat in the body,

- ADVICE - presenting treatment options that can be applied to a specific patient,

- AGREE - obtaining the patient's consent for the proposed therapeutic goal and treatment plan,

- ASSIST - supporting the patient in the therapeutic process [24].

The basis of treatment of overweight and obesity is to obtain a negative energy balance by changing eating habits and increasing physical activity. However, it should be remembered that in patients with an emotional background of increased food consumption, nutritional education itself may be counterproductive; we should remember that such a patient really wants to do this but is unable to. Their brain calls to eat and the fact that he/she does not follow the recommendations is not his/her fault. The role of the doctor is to help such patients through the use of appropriate pharmacotherapy, in some cases also prescribing psychotherapy.

Surgical treatment in patients with an emotional background of increased food consumption should be recommended with caution, and pharmacological and psychological support should be taken into account both during the preparation for surgery and after its performance [25].

\section{Individual selection of pharmacotherapy based on the mechanisms of drug action and the causes of obesity - monotherapy positioning, use of polytherapy}

Currently, there are three drugs available in Poland to support the treatment of overweight and obesity:

- orlistat (gastrointestinal lipase inhibitor) is used only in the group of patients who prefer fat- 
ty foods and have no problems controlling the amount of food consumed (it does not affect the feeling of satiety and appetite). In view of current knowledge on the importance of emotions in the regulation of food intake, this drug is of marginal importance and therefore has not been included in the current guidelines;

- a combination medicinal product containing two active substances: bupropion hydrochloride and naltrexone hydrochloride, reduces food intake by increasing satiety and inhibiting eating under the influence of emotions (appetite). This drug acts synergistically on the neurons of the arcuate nucleus of the hypothalamus. The active substances contained in the drug increase the secretion of proopiomelanocortin of the $\alpha$-melanotropin precursor $(\alpha-\mathrm{MSH})$ (bupropion - stimulation of the feeling of satiety) and prolong its release by blocking the mi opioid receptor (naltrexone - prolonging the feeling of satiety). Moreover, bupropion, by inhibiting dopamine reuptake in the synapses of the mesolimbic reward system, and naltrexone, by blocking opioid receptors, reduce appetite. Thus, this drug acts on both the biological and emotional mechanisms of food intake, which facilitates the patient's implementation of recommendations regarding changes in eating habits $[15,26]$;

- liraglutide, a long-acting GLP-1 receptor analogue, increases the glucose-dependent insulin secretion from pancreatic $\beta$-cells $(1.8 \mathrm{mg} /$ day used in the treatment of type 2 diabetes). Moreover, liraglutide, acting on the centres of satiety and hunger in the hypothalamus, stimulates the feeling of fullness and inhibits the feeling of hunger. This drug does not affect food intake related to emotions; that is the appetite [27].

In accordance with the recommendations of the American Endocrine Associations, short-term (3-6 months) pharmacological treatment of obesity has not shown long-term health benefits in clinical trials and is not recommended. It is also recommended that chronic use of pharmacotherapy be considered due to the chronic nature of the disease. The selection of pharmacotherapy should be individualised [20]. The current guidelines also recommend this approach to the use of pharmacotherapy supporting the treatment of obesity.

We recommend that pharmacotherapy be offered to everyone who is overweight or obese if there are no contraindications to its use.

It is worth remembering that WHO diagnostic criteria based solely on BMI values do not properly reflect the stage of the disease. The new diagnostic criteria of the American Endocrine Societies of 2016 also take into account the occurrence of complications caused by excess fat in the body [20]. In each case of complications caused by excess body fat, in the treatment of which the causative treatment is to achieve weight loss, and in each case of eating under the influence of emotions or low mood, especially if it is accompanied by low self-esteem, the simultaneous implementation of lifestyle changes and pharmacotherapy should be considered.

Individual selection of pharmacotherapy should be based on the identified an etiological factors that led to the development of the disease. As already mentioned, the causal treatment of obesity is the basis for the effective treatment of its complications. The archaic approach of putting the complications of obesity rather than its causes in the selection of pharmacotherapy should be abandoned. Failures in obesity treatment are the result of this approach. Current knowledge indicates that in the selection of pharmacotherapy of complications, obesity should be taken into account as a cause, and in the selection of pharmacotherapy of obesity, factors causing a positive energy balance should be taken into account.

Based on the data from available studies on the aetiology of the disease, as well as efficacy and safety, we recommend the following criteria for selecting pharmacotherapy:

1. Due to its mechanism of action, efficacy, and safety, the drug considered as a first-line drug should be a combined preparation containing bupropion and naltrexone (Mysimba ${ }^{\circ}$ ).

2. Liraglutide in a dose of $3 \mathrm{mg}$ (Saxenda ${ }^{\odot}$ ) should be considered as a second-line drug in a situation where eating under the influence of emotions is excluded (reaching for food in situations of experiencing negative and positive emotions and boredom, eating disorders: compulsive eating syndrome, night-time syndrome, addictive eating) and depressed mood or there are persistent contraindications to the use of the first-line drug.

3. The use of polytherapy with a combination product of bupropion and naltrexone and liraglutide should be considered in patients with impaired carbohydrate metabolism with associated emotional eating. In this case, the dose of liraglutide used should be selected individually $(1.8 \mathrm{mg}$ Victoz ${ }^{\oplus}$ or $3.0 \mathrm{mg}$ Saxenda ${ }^{\odot}$ ) depending on whether only type 2 diabetes or obesity is considered as the indication for the use of liraglutide.

4. Liraglutide doses lower than $3.0 \mathrm{mg}$ daily and other active substances not approved for the treatment of overweight and obesity in monotherapy should not be recommended in monotherapy. 


\section{Justification of the above recommendations}

1. Emotional eating is the most common cause of noncompliance with the recommendations for changing eating habits; the patient wants to but cannot. The use of pharmacotherapy, which does not affect the reward system, does not bring the expected results, increases frustration, and promotes eating. Not all emotional eating can be diagnosed as an eating disturbance. Emotions triggering eating can vary in nature, including tension and fear, but also boredom. The prevalence of eating disorders among obese patients is estimated at $40-70 \%$, but this can be greatly underestimated because they are rarely diagnosed.

2. Both drugs act centrally, but only the combination of bupropion with naltrexone, by acting on the reward system, suppresses appetite and thus eating under the influence of emotions, additionally improves the mood [28]. Isolated studies suggesting an effect of liraglutide on the reward system come from animal studies. In a study using functional magnetic resonance imaging in a group of 20 patients treated for five weeks with liraglutide $5.0 \mathrm{mg}$ or placebo no differences in the response of reward system areas to food images were found between the study groups [29]. The choice of the combination of bupropion and naltrexone as the first-line drug proposed in this document and liraglutide as the second-line drug is not a common recommendation in many existing documents and expert group positions. It may therefore arouse polemics on the part of other experts. However, this position proposes such a prioritization of these drugs also due to the greater acceptance of the form of oral treatment (first-line treatment) than of subcutaneous injections (second-line treatment) and the fact that obese patients more often require therapy affecting the function of the reward system, which helps in dealing with situations where emotions are the cause of consuming excess energy. In addition, experts suggested similar solutions (firstline drug - Mysimba $^{\oplus}$, second-line drug — Saxenda) recently adopted in the current treatment and reimbursement regimens in Norway and Denmark. Perhaps, the emergence of other effective oral medications for the treatment of obesity, as well as new research, will require our opinion to change in the future.

3. The combination of bupropion with naltrexone has a better safety profile. A comparative analysis showed that the number needed to harm (or the number of people in treatment during the year who experience side effects) is 1 in 17 with bupropion and naltrexone in combination and 1 in 4 with liraglutide. Therefore, side effects occur more than four times often during the use of liraglutide [30].

4. Efficacy: a total of 12,868 patients participated in the clinical trials of bupropion and naltrexone combination COR III phase and LIGHT IV phase, with a weight loss of at least $5 \%$ at baseline in $53-80 \%$ (depending on the study) for 56 weeks, and $10 \%$ in 26-55\% [31-35]. In contrast, the phase III SCALE trials, which involved a total of 5358 patients for 56 weeks, achieved a weight loss of at least $5 \%$ at baseline in $63.2 \%$ of patients treated with $3.0 \mathrm{mg}$, and $10 \%$ in $33.1 \%$ [36-39].

5. The cardioprotective effect of liraglutide and the reduction of the risk of death from cardiovascular causes were observed only in the group of patients with type 2 diabetes (LEADER study) [40]; there were no such data in other obesity groups. The LEADER study used the approved dose of liraglutide for diabetes $(1.8 \mathrm{mg} /$ day), not the approved obesity dose $(3.0 \mathrm{mg})$. The results of these studies cannot be extrapolated to the entire population, nor the fact that liraglutide prolongs life expectancy. The LEADER study included patients with known cardiovascular disease and people with cardiovascular risk factors. Subgroup analysis suggests that the cardiovascular benefit of liraglutide was primarily in the subgroup of patients with known cardiovascular diseases in which specific cardiovascular benefits were previously seen. In contrast, a study to evaluate lixisenatide in acute coronary syndrome (ELIXA) showed no cardiovascular benefit from its use in patients with type 2 diabetes and recent acute coronary syndrome. In the ELIXA study, no such group differences with regard to cardiovascular medications were found. Perhaps some of the cardiovascular benefits that were observed in the LEADER study were due to differences in standard of care rather than the use of liraglutide. Multivariate analyses should be carried out, which would at least partially explain these doubts [41]. Post hoc calculations, which are based on the actual sample size, indicate that the sample power for the observed superiority was $75.5 \%$, which is well below the previously planned $90 \%$ power for non-worse. Thus, this lack of power raises concerns about the type I error of superiority [42]. The reduction in $\mathrm{HbA}_{1 \mathrm{c}}$ levels with liraglutide was modest and other factors, such as weight loss, may have contributed to the benefits seen with liraglutide [43]. A high propor- 
tion of patients enrolled in these studies had previously experienced a CVD adverse event; therefore, this therapy is assessed mainly as a secondary prevention of subsequent CVD events. This cannot be fully translated into primary prevention. These studies provide reassuring evidence that the new glucose lowering drugs do not adversely affect CVD events, and some of these drugs may reduce their risk [44]. In the LEADER study, the primary endpoint incidence was $13.0 \%$ lower with liraglutide than with placebo. There was also a $22 \%$ lower CVD death rate and a 15\% reduction in allcause deaths. The number of patients needed to treat (NNT) to prevent one cardiovascular event in three years was 66 and to prevent death from any cause was 98 [45]. It has also been shown that the benefits of liraglutide appear to be limited to a predetermined subgroup of people with known cardiovascular disease. Therefore, the conclusions and implications of the LEADER study are currently limited to the group with type 2 diabetes at high cardiovascular risk [46]. Experts emphasize that our comments and doubts concern only the unauthorized extrapolation of the LEADER study results to the obese patient population. However, they do not undermine the importance of this study for contemporary diabetology and current antidiabetic treatment algorithms, especially in patients at high cardiovascular risk.

6. There are no comparative studies assessing the time it takes for the drugs to take effect.

\section{The use of telemedicine techniques in the treatment of obesity in the pandemic era and in the future}

Currently, in providing remote advice, telephone contact or instant messaging is most often used. However, more and more often telemedicine applications using artificial intelligence algorithms are used. Such solutions are already used in psychiatry. Psychiatrists were also the first to provide recommendations for tele-visiting during a pandemic. According to these recommendations, the conversation with the patient should begin with the assessment of the patient's anxiety related to the epidemic, reassuring him/her and supporting him/her in drawing appropriate conclusions and correcting the information chaos he/she was subjected to. After the patient has calmed down, move on to his/her current health problem. Questions should not be left unanswered, and the patient should be able to contact remotely if necessary [47].
The sanctioning of the place of tele-visiting in the NHF system allowed help to be provided for many patients during the closure of health care facilities due to the pandemic, it also facilitated the introduction of e-prescriptions.

The combination of traditional counselling and televisiting can be an effective tool for diagnosing and treating obesity when there is no time at the clinic. Moreover, the inclusion of telemedicine in the care of obese patients may contribute to the improvement of the doctor-patient relationship and the patient's compliance with the recommendations, including those related to pharmacotherapy. Prior to March 2020, GPs provided sporadic telephone consultations. Before 2019, this type of advice was not sanctioned by the National Health Fund. Medical tele-visit was introduced by the Regulation of the Minister of Health of October 31, 2019, amending the regulation on guaranteed benefits in the field of primary health care Journal of Laws 2019 item 2120. The provisions came into force on November 5 , 2019. The regulation specifies the issue of providing services in outpatient conditions, but also as part of night health care, indicating that remote assistance is possible via teleinformation systems or telephone communication. Thanks to this, the patient has the right to tele-visit.

From January 2020, at least once a year, the family doctor should take anthropometric measurements and assess the nicotine status of each patient reporting to the primary health care practice (new requirement of the National Health Fund - Order of the President of the National Health Fund No. 177/2019 / DSOZ).

Currently, in 2020, the National Health Fund additionally finances the care of patients chronically suffering from diabetes, cardiovascular diseases, or thyroid gland conditions. Most of these patients are also obese. It is advisable that obesity should also be included as a chronic disease in the list of causes of medical consultations qualifying for additional financing by the National Health Fund. Until the new regulations are in place in the GP surgery, advice on obesity may be part of the follow-up advice on chronic diseases. A family doctor who has appropriate knowledge about obesity and drugs supporting its treatment has an ethical duty to treat obesity and propose pharmacotherapy to the patient. In order to increase the knowledge of doctors, current guidelines have been developed.

During the pandemic, tele-visit were also used and reimbursed in specialist counselling.

Telemedicine in the form of the use of medical applications in the treatment of obesity is a new formu- 
la for supporting behavioural and pharmacological therapy. These applications can support the doctor both in the diagnostic process and in making therapeutic decisions, and support treatment through various forms of increasing therapeutic adherence, digital forms of treatment, and monitoring the patient's condition between meetings or tele-meetings with the doctor. Contact between a doctor, dietitian, or psychologist with the patient should be in the form of video-visits, enabling the establishment of eye contact, assessment of the patient's facial expressions and pantomimics as well as establishing contact and a therapeutic relationship.

Telemedicine applications can be particularly useful in increasing therapeutic adherence. Low therapeutic adherence of obesity patients is the cause of incomplete treatment effectiveness, side effects of drugs, and in the long term, somatic and psychological complications of obesity, hospitalisation, and increased treatment costs. The reason for low therapeutic adherence is usually false and inadequate beliefs about obesity, rationalising and contradicting it, beliefs about the ineffectiveness of drugs or the presence of their side effects, or the lack of patient resources (e.g. lack of economic resources to buy drugs or forgetting to take a drug). All these reasons should be taken into account when creating remote telemedicine obesity treatment algorithms.

Telemedicine applications use various methods to support therapeutic adherence that can be used in the treatment of obese patients. Those are:

- reminders in the form of text messages and smartphone notifications,

- video education on the disease and drugs,

- obesity support groups in communities in the Internet environment,

- point systems that strengthen the habit of taking medication,

- video meetings with a doctor, psychotherapist, or dietician.

Improving drug compliance and the effectiveness of obesity treatment using telemedicine tools requires a comprehensive approach to the entire treatment process, not only focusing on therapeutic adherence. In constructing telemedicine obesity therapy, it is advisable to take into account the following recommendations:

Creation and development of user-friendly telemedicine applications enabling full visual-auditory and safe video-connection with the patient.

Creation, research, and popularisation of telemedicine applications for effective stress reduction as a supporting method in the treatment of obesity. Medical applications can thus offer an alternative strategy to deal with negative emotions in relation to food. In reduction of stress in patients treated for obesity can use virtual reality, augmented reality, breathing training, therapeutic games, and prohealth musical frequencies.

Such applications offer great hope for more effective obesity treatment in the near future.

\section{Summary}

Treating obesity in the pandemic era is more important than ever. The current situation is conducive to the development of new diseases and disease worsening, mainly as a result of compensating for negative emotions by eating. Taking into account the data on the impact of obesity and its complications on the severity of the course and the risk of death due to COVID-19, we recommend using the 2016 American Endocrine Society's criteria for the diagnosis of obesity instead of the $1998 \mathrm{WHO}$ criteria. We also recommend diagnosis of eating under the influence of emotions and the occurrence of eating disturbances such as compulsive eating syndrome, night eating syndrome and addictive eating, and the occurrence of obesity complications. It is also advisable to diagnose family members towards overweight and obesity and to involve them in helping the patient.

The approach to treatment should be individualised and should not be limited to nutritional and physical activity education. Each patient should be offered appropriately selected pharmacotherapy, and, if necessary, also psychotherapy. The first-line drug should be a combined preparation containing naltrexone and bupropion $\left(\mathrm{Mysimba}^{\circ}\right)$. Liraglutide in a dose of $3 \mathrm{mg}$ (Saxenda ${ }^{\odot}$ ) should be considered as a second-line drug in a situation where eating under the influence of emotions is excluded (reaching for food in situations of experiencing negative and positive emotions and boredom, eating disturbances: compulsive eating syndrome, night eating syndrome and addictive eating), depressed mood, or there are persistent contraindications to the use of the firstline drug.

It is unethical not to treat obesity or refer the patient to another doctor who will treat it. The use of telemedicine tools can facilitate work in therapeutic teams (doctor, dietitian, psychotherapist), as well as improve patient compliance with pharmacotherapy recommendations and changes in eating habits and the level of physical activity. To facilitate the application of the above guidelines in practice, an algorithm for the diagnosis and treatment of obesity using telemedicine techniques has been developed (Fig. 1). 


\section{Office visit}

- measure body mass and height, calculate BMI, measure waist circumference - if the BMl is $\geq 25 \mathrm{~kg} / \mathrm{m}^{2}$, take a history of obesity complications, or, if necessary, order a diagnosis (their list is attached to the algorithm)

- make a diagnosis based on the recommendations of the American Endocrine Societies

- diagnose eating under the influence of emotions (see the questions in the appendix) and eating disorders (diagnostic criteria in the appendix to the algorithm) and depressed mood (Beck's scale)

\section{Teleadvice}

Ask the patient to weigh him/herself, give his/her weight and height, and calculate BMI:

- if the BMI is $\geq 25 \mathrm{~kg} / \mathrm{m}^{2}$, take a history of obesity complications or, if necessary, order a diagnosis (their list is attached to the algorithm)

- make a diagnosis based on the recommendations

of the American Endocrine Societies

- diagnose eating under the influence of emotions (see the questions in the appendix) and eating disorders (diagnostic criteria in the appendix to the algorithm) and depressed mood (Beck's scale)

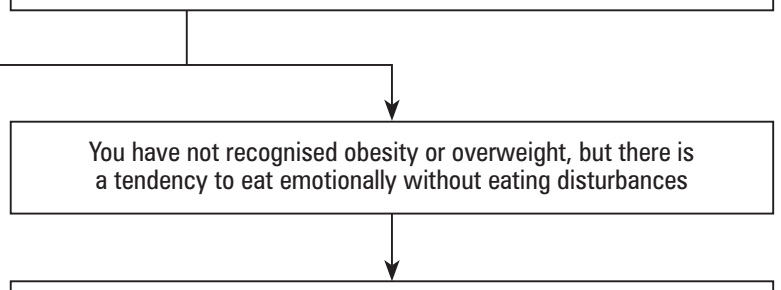

\section{Establish partial and long-term treatment goals} (see recommendation text)

Choose treatment methods individually:

- recommend using the application to monitor food consumption and the level of physical activity

- propose contact using telemedicine tools or visiting the office in order to obtain support in difficult situations

- offer education about changes in eating habits and physical activity through tele-visit or office visits

- propose pharmacotherapy: as a first-line drug, order a combined product containing bupropion and naltrexone (Mysimba ${ }^{\circledR}$ ): in case of contraindications, consider using the second-line drug liraglutide at a dose of $3 \mathrm{mg}\left(\right.$ Saxenda $\left.{ }^{\circledR}\right)$ - see the wording of the recommendation

- obtain the patient's acceptance as to the proposed therapeutic methods

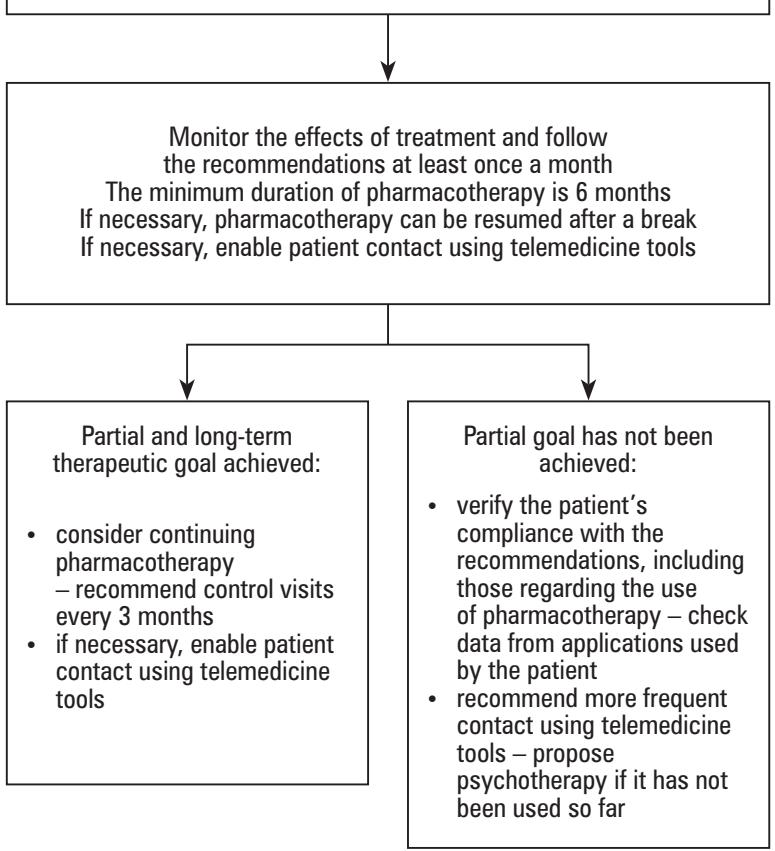

\section{Choose treatment methods individually:} and the level of physical activity

- propose contact using telemedicine tools in order to obtain support in difficult situations

- monitor the patient's body mass once every three months and react in the event of changes, assessing the intensity of eating under the influence of emotions and whether or not eating disturbances develop

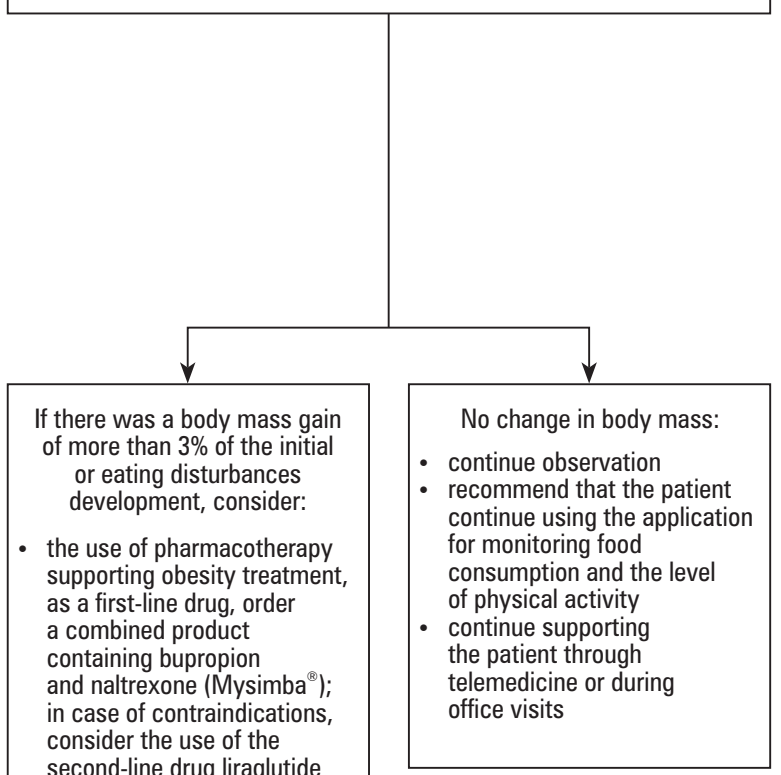

second-line drug liraglutid
- recommend using the application to monitor food consumption

at a dose of $3 \mathrm{mg}$ (Saxenda ${ }^{(1)}$

- see the recommendation

- continue supporting

the patient through

telemedicine or during

office visits

Figure 1. Algorithm for diagnosing and treating obesity using telemedicine techniques 
List of obesity complications that should be included in the history or their diagnosis should be implemented, if not previously performed: prediabetes, type 2 diabetes, dyslipidaemia, hypertension, cardiovascular disease, non-alcoholic fatty liver disease, polycystic ovary syndrome, impaired fertility in women, hypogonadism in men, asthma, sleep apnoea syndrome, hypoventilation syndrome, gastroesophageal reflux disease, stress urinary incontinence, osteoarthritis, depression

Eating under the influence of emotions: reaching for food during or after a stressful situation caused by both positive and negative factors, eating when feeling anxious, rewarding yourself with food, eating when something has failed — comforting with food, eating in situations of boredom, eating in in order to reduce the feeling of fatigue

Ask the patient: Do you feel stomach suction in stressful or anxious situations? Does stress make you reach for food? Do you feel like eating after a stressful situation? Success is food? When something has not turned out, do you reach for food? When you are bored, do you reach for food? Do you use food during other activities, e.g. reading, watching TV, working? Do you reach for food while using the computer? When you feel tired, does eating help to reduce this feeling?

Binge eating syndrome: repeated episodes of unrestrained eating at least once a week for three months, and at least three of the following symptoms: eating much faster than normal, eating until you feel uncomfortably full, eating large amounts of food without feeling physically hungry, eating alone due to embarrassment/embarrassment in eating, feeling disgusted with yourself, depressed or guilty after overeating, as well as marked suffering from eating habits and lack of compensatory activities associated with it (inducing vomiting, using diuretics, increasing physical activity significantly)

Night eating syndrome: eating min. $25 \%$ of the daily food ration after the evening meal or at night with awareness at least twice a week for at least 3 months and at least three of the following symptoms: skipping breakfast due to lack of appetite at least 4 times a week, a strong need to eat between the evening meal and falling asleep or at night, difficulty falling asleep or waking up from sleep at least 4 nights a week, the conviction that food is needed as a condition for starting or returning to sleep, frequent worsening of mood in the evening, as well as significant suffering or deterioration of functioning and lack of criteria for psychological bulimia and the binge eating syndrome

Addictive eating: eating more or more than intended, persistent desire to eat or unsuccessful attempts to limit food consumption, devoting a lot of time to eating activities, neglecting social responsibilities and activities, eating food despite negative physical, mental, and social consequences, limiting or abandoning due to eating important social, professional or recreational activities, and the occurrence of withdrawal syndrome

Figure 1. Algorithm for diagnosing and treating obesity using telemedicine techniques

We hope that it will be a helpful tool to facilitate everyday work and will result in more patients being properly diagnosed and effectively treated.

\section{References}

1. Obesity. Preventing and managing the global epidemic. Report of a WHO, Geneva 1998.

2. Olszanecka-Glinianowicz M, Markuszewski L. Organizacja opieki zdrowotnej nad chorymi na otyłość i zagadnienia etyczne. W: Olszanecka-Glinianowicz M (red.). Obesitologia kliniczna. Med Prakt 2020 [in press].

3. Craig H, le Roux C, Keogh F, et al. How ethical is our current delivery of care to patients with severe and complicated obesity? Obes Surg 2018; 28: 2078-2082.

4. ACOG Committee Opinion No. 763: Ethical considerations for the care of patients with obesity. Obstetr Gynecol 2019; 133: e90-e96.

5. Arora M, Barquera S, Farpour, et al. Stigma and obesity: the crux of the matter. Lancet 2019; 4: 549-550.

6. Zheng KI, Gao F, Wang XB. Letter to the Editor: Obesity as a risk factor for greater severity of COVID-19 in patients with metabolic associated fatty liver disease. Metabolism 2020; 108: 154-244.

7. Muscogiuri G, Pugliese G, Barrea L, et al. Commentary: Obesity: The "Achilles Heel" for COVID-19? Metabolism 2020; 108: 154- 251.

8. Moriconi D, Masi S, Rebelos E, et al. Obesity prolongs the hospital stay in patients affected by COVID-19, and may impact on SARSCOV-2 shedding. Res Clin Pract 2020; 14: 205-209.

9. Petrilli CM, Jones SA, Yang J, et al. Factors associated with hospital admission and critical illness among 5279 people with coronavirus disease 2019 in New York City: prospective cohort study. BMJ 2020; 369: $\mathrm{m} 1966$
10. Chao JY, Derespina KR, Herold BC, et al. Clinical characteristics and outcomes of hospitalized and critically ill children and adolescents with coronavirus disease 2019 (COVID-19) at a Tertiary Care Medical Center in New York City. J Pediatr 2020; S0022- 3476: 30580-30581.

11. Stefan N, Birkenfeld AL, Schulze MB, et al. Obesity and impaired metabolic health in patients with COVID-19. Nat Rev Endocrinol 2020; 23: 1-2.

12. Ryan DH, Ravussin E, Heymsfield S. COVID 19 and the patient with obesity — the editors speak out. Obesity 2020; 10: 22808.

13. Tamara A, Tahapary DL. Obesity as a predictor for a poor prognosis of COVID-19: a systematic review. Diabetes Metab Syndr 2020; 14: 655-659.

14. Brooks SK, Webster RK, Smith LE, et al. The psychological impact of quarantine and how to reduce it: rapid review of the evidence. Lancet 2020; 395: 912-920.

15. Nicholls W, Devonport TJ, Blake M. The association between emotions and eating behaviour in an obese population with binge eating disorder. Obes Rev 2016; 17: 30-42.

16. Frey L, Riva M, Grosshans M, et al. "Food addiction" as a possible risk factor for obesity. Praxis (Bern 1994) 2016; 105: 397-404.

17. Schulte EM, Avena NM, Gearhardt AN. Which foods may be addictive? The roles of processing, fat content, and glycemic load. PLoS One 2015; 10: e0117959.

18. Isasi CR, Parrinello CM, Jung MM, et al. Psychosocial stress is associated with obesity and diet quality in Hispanic/Latino adults. Ann Epidemiol 2015; 25: 84-89.

19. Billes SK, Sinnayah P, Cowley MA. Naltrexone/bupropion for obesity: an investigational combination pharmacotherapy for weight loss. Pharmacol Res 2014; 84: 1-11.

20. Garvey WT, Mechanick JI, Brett EM, et al. Reviewers of the AACE/ ACE Obesity Clinical Practice Guidelines. American Association of Clinical Endocrinologist and American College of Endocrinology 
Comprehensive Clinical Practice Guidelines for Medical Care of Patients with Obesity. Endocr Pract 2016; 22: 1-203.

21. American Psychiatry Association. Diagnostic and Statistical Manual of Mental Disorders (DSM-5). Washington, 2013.

22. Lundgren JD, Allison KC, Stunkard AJ. Night Eating Syndrome: Research, Assessment, and Treatment. Guilford Press, 2012.

23. Bieńkowski P, Szulc A, Paszkowski T, et al. Leczenie nadwagi i otyłości — kto, kiedy i jak? Interdyscyplinarne stanowisko Zespołu Ekspertów. Nutr Obes Metab Surg 2018; 5: 1-10.

24. Welzel FD, Stein J, Pabst A, et al. Five A's counseling in weight management of obese patients in primary care: a cluster-randomized controlled trial (INTERACT). BMC Fam Pract 2018; 19: 97.

25. Wyleżoł M, Olszanecka-Glinianowicz M. Position of the bariatric and metabolic surgery section of the polish association for the study on obesity on the use of pharmacotherapy to support the treatment of obesity before and after surgery. Nutr Obes Metab Surg 2017; 4: 1-7.

26. Olszanecka-Glinianowicz M, Filipiak KJ, Narkiewicz K, et al. Stanowisko Zespołu Ekspertów dotyczące zastosowania produktu leczniczego Mysimba (chlorowodorek bupropionu i chlorowodorek naltreksonu) we wspomaganiu leczenia otyłości i nadwagi $\left(\mathrm{BMI} \geq 27 \mathrm{~kg} / \mathrm{m}^{2}\right)$ z chorobami towarzyszącymi. Chor Serca Nacz 2016; 13: 333-348.

27. Nuffer WA, Trujillo JM. Liraglutide: A new option for the treatment of obesity. Pharmacotherapy 2015; 35: 926-934.

28. Guerdjikova AI, Walsh B, Shan K, et al. Concurrent improvement in both binge eating and depressive symptoms with Naltrexone/ Bupropion therapy in overweight or obese subjects with major depressive disorder in an open-label, uncontrolled study. Adv Ther 2017; 34: 2307-2315.

29. Farr OM, Upadhyay J, Rutagengwa C, et al. Longer-term Liraglutide administration at the highest dose approved for obesity increases reward-related orbitofrontal cortex activation in response to food cues: Implications for plateauing weight loss in response to anti-obesity therapies. Diabetes Obes Metab 2019; 21: 2459-2464.

30. MacDaniels JS, Schwartz TL. Effectiveness, tolerability and practical application of the newer generation anti-obesity medications. Drugs Context 2016; 5: 212291 .

31. Greenway FL, Fujioka K, Plodkowski RA, et al. Effect of naltrexone plus bupropion on weight loss in overweight and obese adults (CORI): a multicentre, randomised, double-blind, placebo-controlled, phase 3 trial. Lancet 2010; 376: 595-605.

32. Apovian CM, Aronne L, Rubino D, et al. A randomized, phase 3 trial of naltrexone SR/bupropion SR on weight and obesity-related risk factors (COR-II). Obesity (Silver Spring) 2013; 21: 935-943.

33. Wadden TA, Foreyt JP, Foster GD, et al. Weight loss with naltrexone SR/bupropion SR combination therapy as an adjunct to behavior modification: the COR-BMOD trial. Obesity (Silver Spring) 2011; 19: $110-120$.
34. Hollander P, Gupta AK, Plodkowski R, et al. Effects of naltrexone sustained-release/bupropion sustained-release combination therapy on body weight and glycemic parameters in overweight and obese patients with type 2 diabetes. Diab. Care 2013; 36: 4022-4029.

35. Nissen SE, Wolski KE, Prcela L, et al. Effect of Naltrexone-Bupropion on major adverse cardiovascular events in overweight and obese patients with cardiovascular risk factors: a randomized clinical trial. JAMA 2016; 315: 990-1004.

36. le Roux CW, Astrup A, Fuijoka K, et al. 3 years of liraglutide versus placebo for type 2 diabetes risk reduction and weight management in individuals with prediabetes: a randomised, double-blind trial. Lancet 2017; 389: 1399-1409.

37. Davies MJ, Bergenstal R, Bode B, et al. Efficacy of liraglutide for weight loss among patients with type 2 diabetes: the scale diabetes randomized clinical trial. JAMA 2015; 314: 687-699.

38. Pi-Sunyer X, Astrup A, Fuijoka K, et al. A randomized, controlled trial of $3 \mathrm{mg}$ liraglutide in weight management. N Engl J Med 2015; 373: 11-22.

39. Blackman, A, Foster GD, Zammit G, et al. Effect of liraglutide 3.0 $\mathrm{mg}$ in individuals with obesity and moderate or severe obstructive sleep apnea: the SCALE Sleep Apnea randomized clinical trial. Int J Obes (Lond) 2016; 40: 1310-1319.

40. Marso SP, Daniels GH, Brown-Frandsen K, et al. Liraglutide and cardiovascular outcomes in type 2 diabetes. N Engl J Med 2016; 375: 311-322.

41. Álvarez-Villalobos NA, Treviño-Alvarez AM, González-González JG. Liraglutide and cardiovascular outcomes in type 2 diabetes. New Engl J Med 2016; 375: 1797-1798.

42. Correia LCL, Latado A, Porzsolt F. Liraglutide and cardiovascular outcomes in type 2 diabetes. N Engl J Med 2016; 375: 1798.

43. Doggrell SA. Liraglutide, a GLP-1 receptor agonist, prevents cardiovascular outcomes in patients with type 2 diabetes. Evid Based Med 2017; 22: 28.

44. Bailey CJ, Marx N. Cardiovascular protection in type 2 diabetes: Insights from recent outcome trials. Diabetes Obes Metab 2019; 21: 3-14.

45. Athyros VG, Katsiki N, Tentolouris N. Editorial: Do some glucagonlike-peptide-1 receptor agonists (GLP-1 RA) reduce macrovascular complications of type 2 diabetes mellitus a commentary on the liraglutide effect and action in diabetes: evaluation of cardiovascular outcome results (LEADER) Trial. Curr Vasc Pharmacol 2016; 14: 469-473.

46. Kosiborod M. Following the LEADER - why this and other recent trials signal a major paradigm shift in the management of type 2 diabetes. J Diabetes Complications 2017; 31: 517-519.

47. Krzystanek M, Matuszczyk M, Krupka-Matuszczyk I, et al. List do redakcji. Polskie rekomendacje w zakresie prowadzenia wizyt online w opiece psychiatrycznej. Psychiatr Pol 2020; 54: 391-394. 